\title{
Signal Analysis of a Three Axis Aircraft Stabilized Platform
}

\author{
JING Tai-long ${ }^{1,}$, , QU Sheng-li ${ }^{2, b}$, WANG Hai-bing ${ }^{3, c}$,WEI Dong ${ }^{4, d}$ \\ ${ }^{1,2,3,4}$ No. 2, South Taibai Road Xi'an City, Shaanxi Province, China

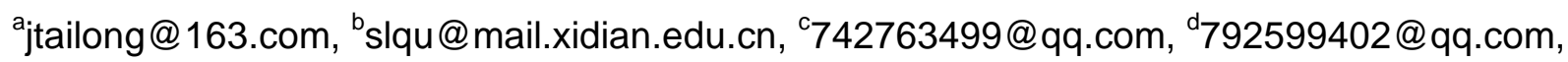

Keywords: Stabilized Platform, noise signal analysis, Multimedia Signal Processing.

Abstract. When an aircraft is on a steady flight, there exists a series of jamming signals, which are detected and recorded by the airborne equipment. By analyzing those signals, the design of a stable platform controller of the aircraft in feed-forward form will be improved. During the analysis of stable flight in time domain, the frequencies of those signals are roughly fetched. Those frequencies are proved to be right through the method in frequency domain. Based on correctness of the precious, the amplitude of those signals are got by the method of Area rule. The phases of those signals will be calculated by the method of correlation. Pairwise compress the composite signal, the simulation match the original signal well.

\section{Introduction}

\section{stabilized platform}

As is shown in figure 1, a stabilized platform is widely used in the zone of enemy investigation, target location, attack collation, photograph and effect evaluation. The aim of stabilized platform is to isolate external disturbance. Thus the position of the platform can stay the same.

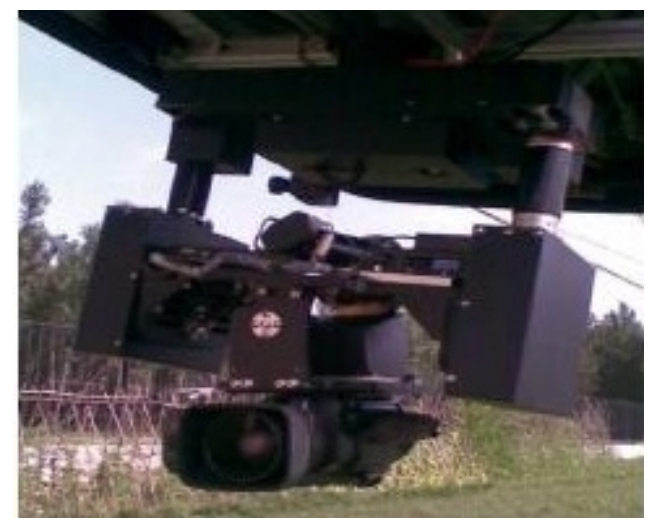

Figure 1: A sample use of stabilized platform

As is showed in picture 2(a), the whole stabilized platform system mainly consists controller, position sensor, three actuating mechanisms and three stories frame structure. The position sensor consists three micromechanical gyroscopes, and three acceleration sensors. Micromechanical gyroscope is used as the feedback element of velocity-loop, and acceleration sensor serves as the feedback element of position loop. Actuating mechanism is realized by DC servo motor. From picture 2(b), the loops of three-axis stabilized platform are azimuth loop, roll loop and pitch loop. The cross point of three axis is immovable. DC servomotors drive the rotors move, and then act on the three loops. 


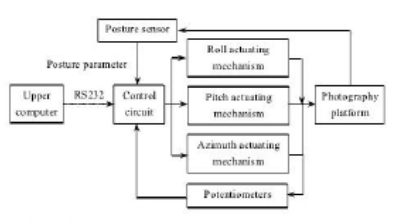

(a) Block diagram of system principle

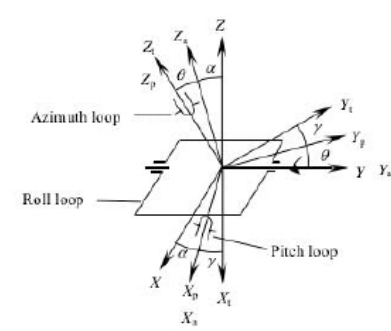

(b) Frame structure of stabilized platform and coordinates graph

Figure 2: Basic principium of the platform

\section{noise signal}

Noise is a factor that influences the stability of the platform, which will impact the development of aerial photograph and robot vision, etc. The analysis of signals will promote the develop of controllers design for the platform. In the work [1], the telemetry signal is usually single channel signal. And when analyzing the time series of the flight test process, it takes the stationary stage signal as the reference noise, and uses it to process the feature stage signal for noise cancellation, which means the method doesn't fit our more complicated situation. In the work [2], basic principle and method of wavelet and wavelet packet analysis are elaborated. On this background, it introduces the general method steps of making a de-noising for signal by using of wavelet packet method. But, the noise signal existing in our stabilized platform is continuous. In the work [3], it searches the optimal frequency shift. But the white noise is continuous. In the work [17], it analyzed multi-source noise components and studies the partial correlation analysis. When the noise signals can't be canceled, the method won't work. In the work [4], it just analyzes the additive white Gaussian noise. In the work [5], the frequencies of the signals have been given. So, it can fit our situation when the signals frequencies are unknown.

In this article, we combine time domain and frequency domain to confirm the element of the signal roughly, use the method of area proportion to confirm the amplitude, and confirm the phase position by means of co-correlation. Without changing the original signal, the composite signal mixed with white noise matches well, which proves the method we use is right.

\section{Data analysis}

Through MATLAB, the data recorded during the flight will be analyzed in time domain. By analyzing the figure 3, we cut the data into three phase: launch $(0 \mathrm{~s}-620 \mathrm{~s})$, stable stage (620s-2652s), landing (2652s-4056s).

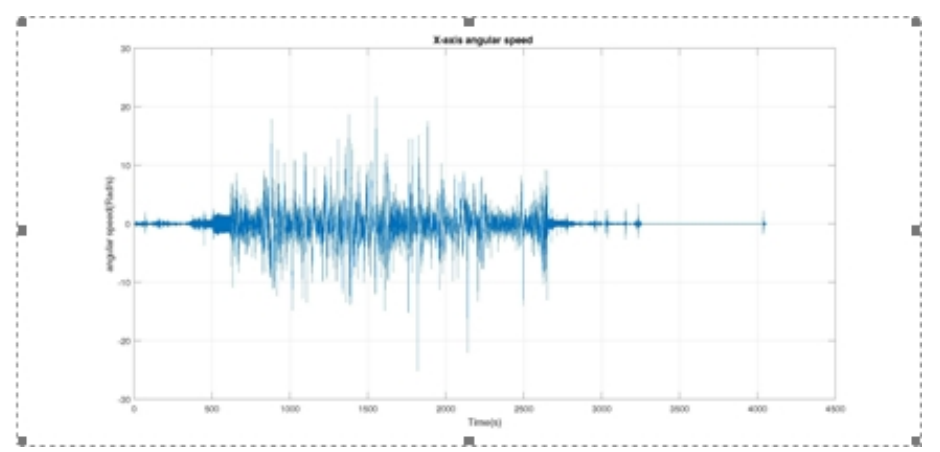

Figure 3: X-axis angular speed

Here, as is showed in figure 4, we dispose the 'stable stage' alone. 


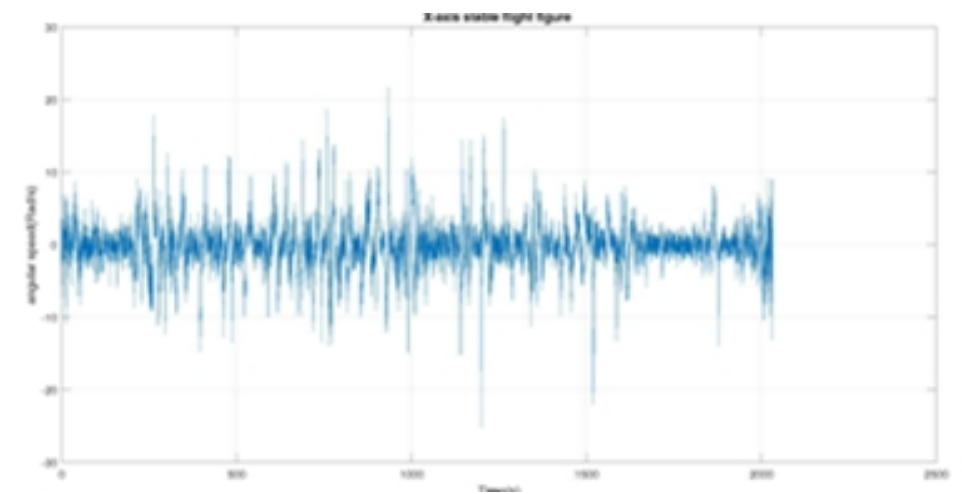

Figure 4: $\mathrm{X}$-axis stable flight figure

\section{signal decomposition}

By observing and magnifying the figure above in time domain, we can analyze the signal consists of a series of sinusoidal signals. Based on this, we estimate the frequency of the sinusoidal signals roughly by magnifying the angular rate graphic of the stable stage.

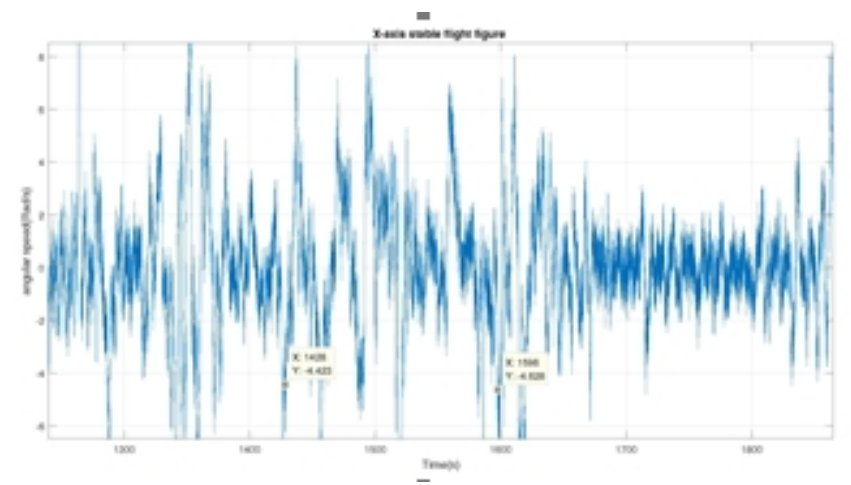

Figure 5: sample of means to get frequency

As showed in figure 5, there exist some sinusoidal signals. During the marked time, we can calculate a kind of sinusoidal signal including 5 periods. So, the frequency is:

$$
f=\frac{1}{T}=\frac{1}{\frac{1306-1015}{9}} \mathrm{~Hz}=0.03 \mathrm{~Hz}
$$

Through this method, we can get other frequency of the signals $0.62 \mathrm{~Hz}-18.5 \mathrm{~Hz}$.

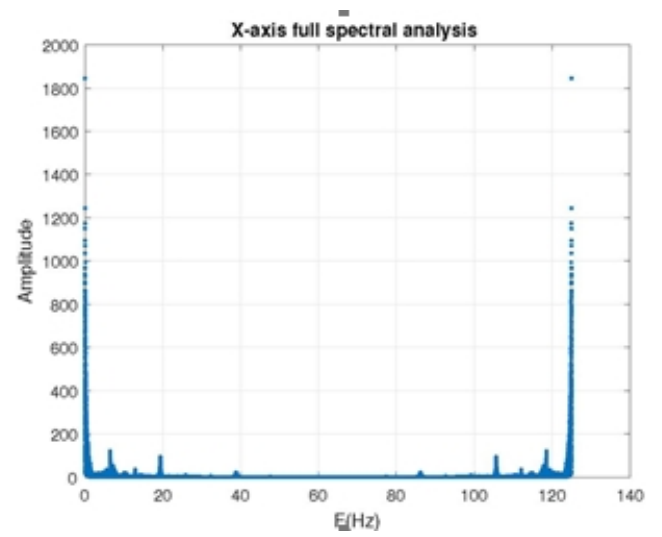

Figure 6: $\mathrm{X}$-axis full spectral analysis 
From the picture 6, it shows that there exists a signal of $0.03 \mathrm{~Hz}$, which matches the precious result. Also, the signal intensity below $1 \mathrm{~Hz}$ is powerful. So we consider that there exist some low-frequency sinusoidal signals between $0.03 \mathrm{~Hz}$ and $1 \mathrm{~Hz}$, which have the similar strength and the frequency equals to $0.4 \mathrm{~Hz}$.

So, here is the list of the signals:

decrement signals
$0.03 \mathrm{~Hz}$
$0.4 \mathrm{~Hz}$ a series of signals
$6.47 \mathrm{~Hz}$
$12.93 \mathrm{~Hz}$
$19.4 \mathrm{~Hz}$
$38.8 \mathrm{~Hz}$
$47.65 \mathrm{~Hz}$

\section{amplitude $A$ calculation}

Assume that equation 2 is a sin signal including frequency of $0.03 \mathrm{~Hz}$, whose envelope line with $x$-axis produce an area around $0.03 \mathrm{~Hz}$ recorded as $s_{1}$; while equation 3 process Fourier transform in sampling frequency of $125 \mathrm{~Hz}$, and the area is marked as $s_{0}$.

$$
\begin{aligned}
& y_{1}=A \sin \left(2 \pi 0.03 t+\varphi_{2}\right) \\
& y_{2}=A \sin (2 \pi 0.03 t)
\end{aligned}
$$

then

$$
A=\frac{s_{1}}{s_{2}} \quad A=0.2371
$$

We can calculate the other frequencies' $A$ by using the same method.

\begin{tabular}{ccc}
\hline$f$ & $A$ & $x(n)$ \\
0.03 & 0.1558 & $0.1558 \sin (2 \pi \times 0.03 t)$ \\
0.4 & 0.0582 & $0.0582 \sin (2 \pi \times 0.4 t)$ \\
6.47 & 0.1002 & $0.1002 \sin (2 \pi \times 6.47 t)$ \\
12.93 & 0.0093 & $0.0093 \sin (2 \pi \times 12.93 t)$ \\
19.4 & 0.0664 & $0.0664 \sin (2 \pi \times 19.4 t)$ \\
38.8 & 0.0089 & $0.0089 \sin (2 \pi \times 38.8 t)$ \\
47.65 & $4.7824 \times 10^{-5}$ & $4.7824 \times 10^{-5} \sin (2 \pi \times 47.65 t)$ \\
\hline
\end{tabular}




\section{calculation $\varphi$}

relevance proof of a conclusion

If, two sequence as shown in equation 4 , while $\eta_{x}(n)$ and $\eta_{y}(n)$ are two independent noise signals.

$$
\begin{aligned}
& x(n)=s_{1}(n)+\eta_{x}(n)=\alpha \cdot e^{j\left(\omega n+\theta_{x}\right)}+\eta_{x}(n) \\
& y(n)=s_{2}(n)+\eta_{y}(n)=\beta \cdot e^{j\left(\omega n+\theta_{y}\right)}+\eta_{y}(n)
\end{aligned}
$$

Introducing reference signal $z(n)=e^{j \omega n}$ mutually correlates $x(n)$ and $y(n)$ respectively.

$r_{x z}(n)=E[x(n) z(n)]=E\left\{\left[s_{1}(n)+\eta_{x}(n)\right] z(n)\right\}=E\left\{s_{1}(n) z(n)+\eta_{x}(n) z(n)\right\}=r_{s_{1} z}(n)+r_{\eta_{x} z}(n)$

In equation $5, \eta_{x}(n)$ is independent of $z(n)$. Thus

$$
r_{\eta_{x} z}(n)=0
$$

then

$$
r_{x z}(m)=r_{s_{1} z}(m) n=E\left[\alpha \cdot e^{-j\left(\omega n+\theta_{x}\right)} e^{j \omega n}\right]=\alpha \cdot e^{-j \theta_{x}}
$$

therefore

$$
r_{y z}(m)=r_{s_{2} z}(m)=E\left[\alpha \cdot e^{-j\left(\omega n+\theta_{y}\right)} e^{j \omega n}\right]=\alpha \cdot e^{-j \theta_{y}}
$$

By combining equations 6 and 7

$$
\frac{r_{y z}(m)}{r_{x z}(m)}=\frac{\beta}{\alpha} e^{j\left(\theta_{x}-\theta_{y}\right)}
$$

then,

$$
\theta_{x}-\theta_{y}=\arg \frac{r_{y z}(m)}{r_{x z}(m)}
$$

\section{Application in our case}

let $f=0.03 \mathrm{~Hz}$ for instance, there will be

$$
x(n)=0.1558 \sin (2 \pi \times 0.03 t)+\eta(t)
$$

$\eta(t)$ is a noise signal.

$$
y(n)=0.1558 \sin (2 \pi \times 0.03 t)+\delta(t)
$$


in which, the sampling time of $y(n)$ is $0.008 \mathrm{~s}$. The average value of white noise $\delta(t)$ is 0 , the variance of it is 0.000003 .

$$
z(n)=\sin (2 \pi \times 0.03 t)
$$

then

$$
\varphi=-0.0933
$$

We can find the $\varphi$ of other signals through the same method.

\section{verification}

$$
\begin{aligned}
& x_{1}=0.1558 \sin (2 \pi \times 0.03 t-0.0933) \\
& x_{2}=0.0582 \sin (2 \pi \times 0.4 t-0.0571) \\
& x_{3}=0.1002 \sin (2 \pi \times 6.47 t-0.0933) \\
& x_{4}=0.0093 \sin (2 \pi \times 12.93 t-0.000024051) \\
& x_{5}=0.0664 \sin (2 \pi \times 19.4 t-0.000033886) \\
& x_{6}=0.0089 \sin (2 \pi \times 38.8 t-0.0000082362) \\
& x_{7}=0.0000047824 \sin (2 \pi \times 47.65 t+0.000000047182)
\end{aligned}
$$

let

$$
x=x_{1}+x_{2}+x_{3}+x_{4}+x_{5}+x_{6}+x_{7}+u
$$

In equation 9 , the average of white noise $u$ is 0 , the variance of it is 0.000003 . The comparison between composite signal and the original one is below:

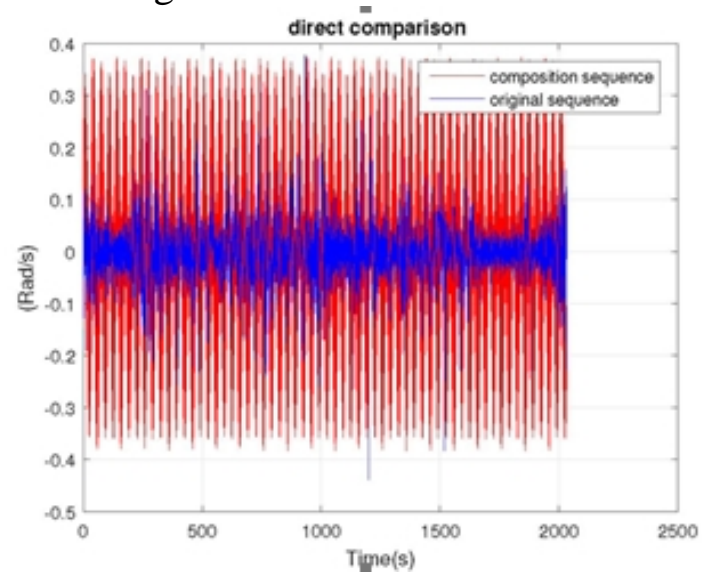

So we consider subsection process of the signal. The coefficient of compressibility is below:

\begin{tabular}{cccc}
\hline & time sharing stage & duration & coefficienty \\
one & $0-250$ & $250 \mathrm{~s}$ & 0.2 \\
two & $250-1675$ & $1425 \mathrm{~s}$ & 0.4 \\
three & $1675-1850$ & $175 \mathrm{~s}$ & 0.1 \\
four & $1850-2032$ & $182 \mathrm{~s}$ & 0.2 \\
\hline
\end{tabular}

Now, the verification will be done step by step. 
During step one, we multiply stage $0-250$ s with 0.2 , the rest holds on.

$$
x_{T_{1}}=0.2 x_{t_{1}}+x_{\Delta 1}
$$

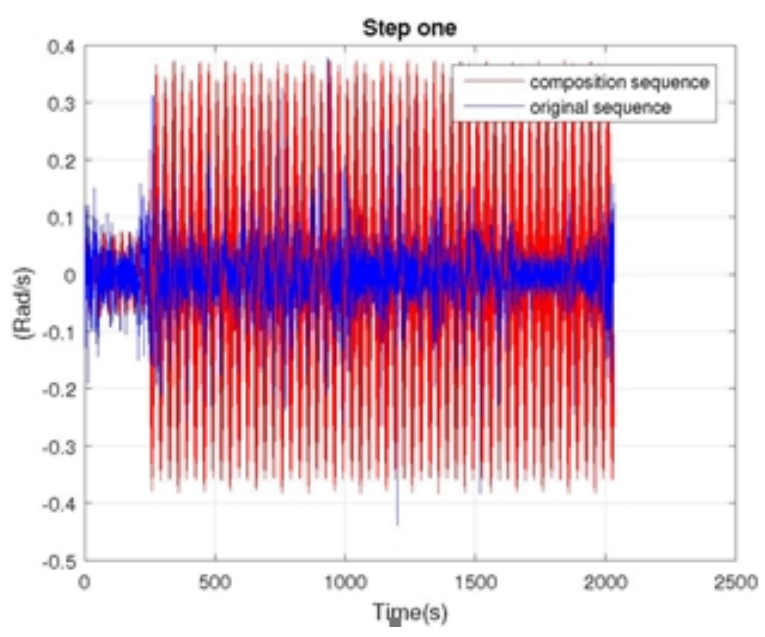

During step two, based on step one, stage 250s-1675s multiplies 0.4. The rest hold on.

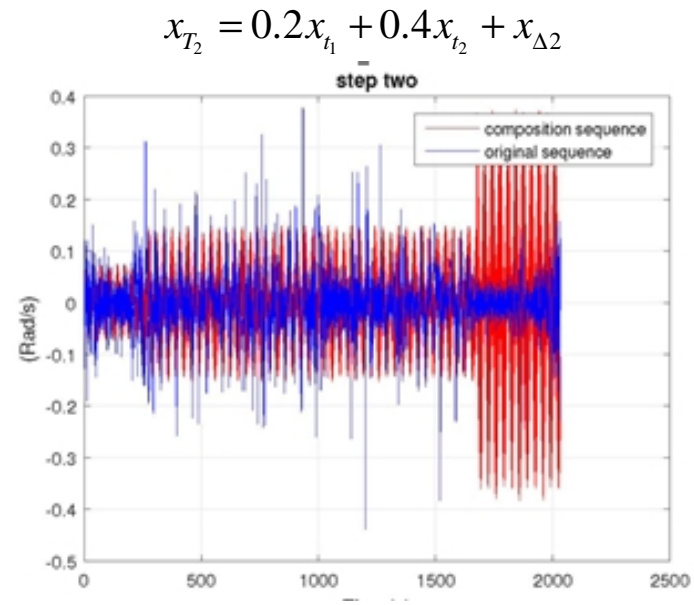

During step three, based on step two, stage 1675s-1850s multiplies 0.1 , the rest holds on.

$$
x_{T_{3}}=0.2 x_{t_{1}}+0.4 x_{t_{2}}+0.1 x_{t_{3}}+x_{\Delta 3}
$$

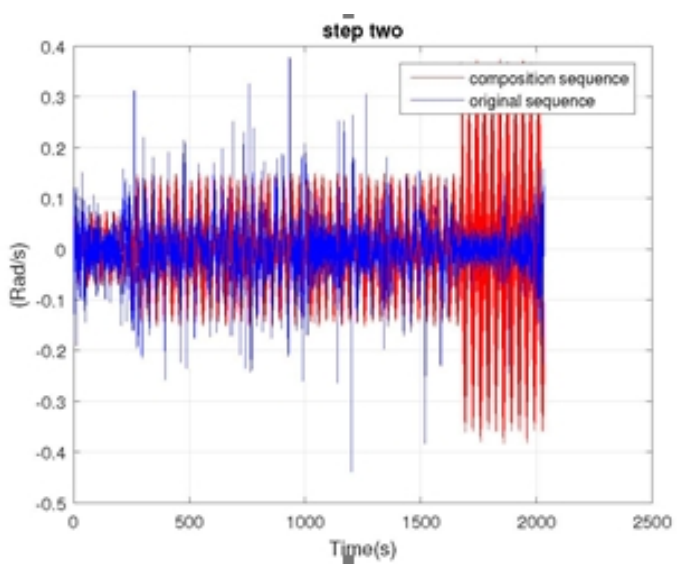

During step four, based on step three, stage $1850 \mathrm{~s}-2032 \mathrm{~s}$ multiplies 0.2 , and we finally get: 


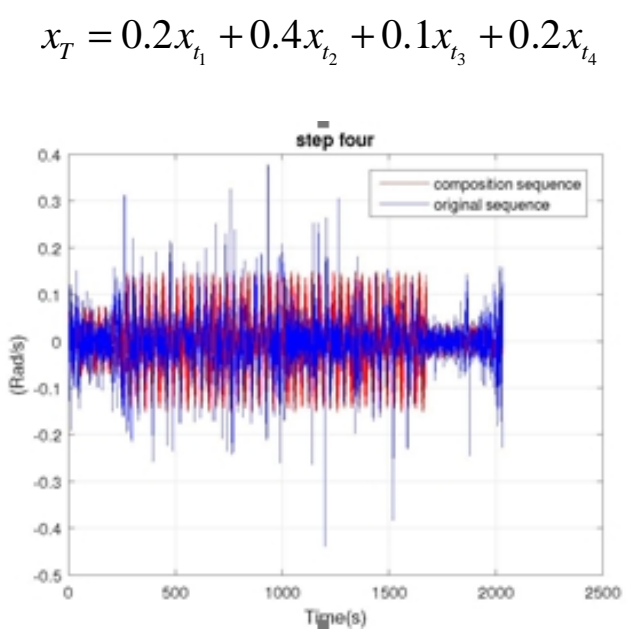

Thus we verify the correction of the method.

\section{Conclusion}

In this article, we propose a method that how to analyze a complex noise signal in a useful way. This method is more effective when used in most engineering applications these don't need high accuracy. Our further study will be focused on how to improve the accuracy of the method.

\section{References}

[1] GUO Feng, "Noise Cancellation Method of the Telemetry Signal Based on Time Series Analysis," 2015 International Conference on Computer Science and Mechanical Automation, 2015, pp. 201-215.

[2] LI Yi-feng, ZHANG Li-hui, and LI Bao-hui etc., "The comparison study of wavelet and wavelet packet analysis in the de-noising of EEG test signal," 7th International Conference on Information Technology in Medicine and Education, 2015, pp. 274-277.

[3] YANG Wen-ge, LU Wei-tao, HONG Jia-cai, and HU Kai, "A Modified Denoising Algorithm for Sinusoid Signals Based on SUWPT," Journal of Spacecraft TT \\& C Technology, vol. 32, no. 5, pp. 378-382, Oct. 2013.

[4] HUANG Chao, SUO Ji-dong, and YU Liang, "A Novel Algorithm for Estimation of Sinusoid Frequency Based on Argument of Sample Autocorrelation Function," Telecommunication Engineering, vol. 54, no. 1, pp. 63-67, Jan. 2014.

[5] ZHANG Xiao-wei, MENG Fan-ming, "Research on estimation of sine signal amplitude and initial phase. Computer Engineering and Applications," Computer Engineering and Applications, vol. 49, no. 5, pp. 216-219, May. 2013.

[6] QIU Bao-mei, WANG Feng-juan, and WANG Jian-wen, "Small Aerial Photography Stabilized Platform Based on Fuzzy-PID Control," Opto-Electronic Engineering, vol. 37, No. 10, pp. 23-28, Oct. 2010.

[7] Sun Gao, Research on Control System of Semi-Strapdown Photoelectricity Stabilized Platform, Changchun Institute of Optics, Fine Mechanics and Physics Chinese Academy of Sciences, pp.63-65, May. 2013.

[8] Cui Maihui, Zhou Jianjun, Chen Chao, and Jiang Yufeng,"Control Loop Design of Shipboard Opto-electronic Imaging Three-axis Independent Stabilized Platform," Computer Measurement $\backslash \&$ Control. vol. 18, pp. 810-812, April. 2010.

[9] CHENG Zhi-feng etc, "Application of high volume fraction SiC/Al composites to unmanned airborne photoelectric platforms," Optics and Precision Engineering. vol. 17, no. 11, pp. 2821-2826, Nov. 2009. 
[10] LI Xian-tao, The Method for Restraint of Destabilization of Airborne Photoelectric Stabilized Platform, Changchun Institute of Optics, Fine Mechanics and Physics Chinese Academy of Science, pp. 45-65, May. 2014.

[11] DING Ce, DAI Ming, LI Jia-quan, and WANG Yong-yang, "The PDF Control of Airborne Photoelectric Gyro Stabilized Platform," Opto-Electronic Engineering, vol. 39, no. 10, pp. 128-134, Otc. 2012.

[12] LI Ming, MA Jun, and ZHOU Xing-yi, "Study of measurement technique on airborne photoelectric stabilized platform," Optics and Precision Engineering, vol. 14, no. 5, pp 847-852, Oct. 2006.

[13] GAN Zhi-hong, ZHANG Bao, and HAN Peng-peng, "Engineering analysis of airborne optoelectronic platform frame structure," Optics and Precision Engineering, vol. 16, no. 12, pp. 2441-2446, Dec. 2008.

[14] LI Zeng-yan, LI Xiao-min, ZHOU Zhao-ying, and LIU Qiu-sheng, "Hardware-in-the-Loop Simulation Method of Airborne Stabilized Platform," Electronics Optics \\& Control, vol. 23, no. 6, pp. 60-63, June. 2016.

[15] SONG Duan-po, XU Jian-zhong, SHE Hong, WANG Wen-jun, and YU Zhu-qing, "Structural Analysis of The Triaxial Airborne Stabilized Platform Based on ANSYS," JOURNAL OF JIANGSU POLYTECHNIC UNIVERSITY, vol. 21, no. 2, pp. 31-33, June. 2009.

[16] CHANG Jiu-jian, NI Tian-rong, and MEI Liang, "Research on Slide Mode Control Method for Airbone Stabilized Platform," Radar Science and Technology, vol. 12, no. 6, pp. 671-674, Dec. 2014.

[17] CHEN Xi-xin, JIANG Guo-jian, and XU Xin-sheng, "A system of analyzing multi-source noise comPonents based on the noise caneeling method," ACTA ACUSTICA, vol. 28, no. 3, pp. 272-276, May. 2003. 